\title{
Transition-State Model for Entropy-Limited Freezing
}

\section{Citation}

Aziz, Michael J., P. S. Peercy, Michael O. Thompson, and Jeff Y. Tsao. 1987. Transition-State model for entropy-limited freezing. Materials Research Society Symposia Proceedings 74:

117-122.

\section{Published Version}

http://www.mrs.org/s_mrs/sec.asp?CID=1727\&DID=38980

\section{Permanent link}

http://nrs.harvard.edu/urn-3:HUL.InstRepos:2848641

\section{Terms of Use}

This article was downloaded from Harvard University's DASH repository, and is made available under the terms and conditions applicable to Other Posted Material, as set forth at http:// nrs.harvard.edu/urn-3:HUL.InstRepos:dash.current.terms-of-use\#LAA

\section{Share Your Story}

The Harvard community has made this article openly available.

Please share how this access benefits you. Submit a story.

\section{Accessibility}




\section{TRANSITION-STATE MODEL FOR ENTROPY-LIMITED FREEZING}

J.Y. Tsao*, M.J. Aziz**, P.S. Peercy* and M.O. Thompson***

* Device Research Dept., Sandia National Labs, Albuquerque, NM 87185

* Div. Of Applied Sciences, Harvard University, Cambridge, MA 02138

** Dept. of Materials Science, Cornell University, Ithaca, NY 14853

\section{ABSTRACT}

A brief review is given of transition-state theory, both for the case of unimolecular reactions in the gas phase, and for reactions in condensed phases. An argument is made that, within the context of this theory, heterogeneous freezing in $\mathrm{Si}$ is limited to rates much lower than collision rates by the difference between the entropies of the solid and the liquid.

\section{INTRODUCTION}

Since its formulation in the $1930^{\circ} \mathrm{s}$ [1], transition-state and its variants have been successful in accounting for a large body of experimental reaction-rate data. For example, for unimolecular reactions in the gas phase, the theory is known to chemical kineticists as the highly successful RRKM theory [2]. For condensed-phase reactions and transformations transition-state theory has also yielded useful results [3], although in these cases it is difficult to deduce from first principles the exact nature of the transition state.

Recently, we compared the predictions of transition-state theory with constraints, both experimentally measured as well as derived from first principles, on the kinetics of heterogeneous melting and freezing of $S i$ [4]. Our conclusion was that if transition-state theory were valid, and if a single mechanism were operative at all temperatures, then the constraints enumerated were only consistent with an "entropy-limited" freezing model, in which the transition state has an enthalpy greater than or equal to that of the liquid, and an entropy less than or equal to that of the solid. In effect, not all collisions of liquid atoms onto the solid/liquid interface result in freezing, but only those which bring the liquid atom into special configurations closely approximating the solid.

More recentiy, measurements indicate that there may be multiple mechanisms for melting and freezing, each of which may be dominant in a different temperature regime [5-7]. A single theory for the kinetics of melting and freezing across the entire temperature range may therefore be inappropriate. Nevertheless, if the mechanisms are independent, it may be possible (and it seems reasonable to attempt) to describe the rates of each mechanism individually using some form of transition-state theory [8].

In the first part of this paper, we review briefly the structure and assumptions of transition-state theory. We begin with the simpler case of unimolecular reactions in the gas phase, and then turn to reactions in condensed phases. In the second part, we argue that for reversible transitions between states having very different enthalpies and entropies, e.g., the solid-liquid transformation in $S i$, transition-state theory is naturally asymetric, i.e., successful forward and backward "hopping" rates are very different [9]. 


\section{REACTIONS IN THE GAS PHASE}

It is instructive to consider (thermal) unimolecular reactions in the gas phase, since in these cases the processes of energization and reaction are clearly separable. The generally accepted overall mechanism [10] involves, first, energization of reactant $R$ by collisions with heat-bath molecules $M[11]$,

$$
R+M \frac{k_{1}}{-\frac{1}{k_{2}}>-} R^{*}+M,
$$

and, second, unimolecular reaction of energized molecules $R^{*}$,

$$
R^{*}-\cdots-\infty R^{+}-\ldots
$$

mediated by transition states $R^{+}$. Assuming the reactant to at least be metastable, the configuration space associated with reactant and product can only be joined across a critical surface having a higher energy than that of the ground-state reactant. Constant-energy trajectories passing through this critical surface react and represent transition states. of the energized molecules, the transition state population is considered to be statistical: the system is represented equally by all points in phase space consistent with a specified energy. Hence, only the gross, statistical properties of the transition states are important in calculating their occupation probabilities.

The forward reaction rate is calculated solely on the basis of the properties of the transition states, without knowledge of the properties of the product. This rate is determined by the energization rate (Eq. 1), in series with the decay rate (Eq. 2), and will naturally depend on the relative rates for the two processes.

In the "high pressure" Iimit, collisional energization/de-energization is so fast that the distribution of reactant states is always thermal. The reaction rate is then given by the decay rates of the transition states, weighted by their thermal occupation probabilities.

In the "low pressure" init, unimolecular decay is so fast that the distribution of reactant states is truncated at the critical energy associated with the transition states. The reaction rate is then given by the energization rate into the transition states. This is equivalent to a collision rate weighted by the thermal occupation probabilities of the transition states calculated in the absence of reaction [12]. In a sense, reactions occuring in the low-pressure limit may be considered "ballistic", in that after each colision molecules either react completely or not at all according to a predetermined phase-space trajectory. 


\section{REACTIONS IN THE CONDENSED PHASE}

In the condensed phase, reactions are conceptually more complex, since many more bodies are involved, and since it is difficult to arrive at a microscopic picture on which to base the thermodynamic properties of the transition state, Consider the reversible reaction $s<-->1$, depicted schematically in Fig. 1. The relevant partition functions can be written as quantum-mechanical sums over states,

$$
\begin{aligned}
& Z_{s}={ } s " \text { states } e^{-E / k T} \text {, } \\
& z_{1}=\text { "ln states } e^{-E / k T} \text {. } \\
& \mathrm{Z}_{\mathrm{S}^{+}}=\mathrm{ns}_{\mathrm{s}+} \sum_{\text {states }} \mathrm{e}^{-\mathrm{E} / \mathrm{kT}}, \\
& \text { and } Z_{1+}=n_{1+n} \sum_{\text {states }} e^{-E / k T} \text {, }
\end{aligned}
$$

from which the thermodynamic quantities $\bar{H}=\bar{E}=k T^{2}[\mathrm{~d} \ln \mathrm{E} / \mathrm{dT}]$ and $S=\overline{\mathrm{H}} / \mathrm{T}+$ klnz can be calculated [13]. Here, $s^{+}$and $I^{+}$are transition states, and are considered, as iliustrated in Fig. 1, to represent special configurations within the starting $s$ and 1 manifolds from which reaction may occur. We suppose, then, that $Z_{s_{+}}$and $Z_{Z_{+}}$are subsets of $Z_{s}$ and $Z_{1}$, respectively. Furthermore, within the context of transition-state theory, direct transitions between $s$ and 1 states which are not part of $s^{+}$or $1+$ are not allowed $[14]$.

In a manner analogous to the case of reactions in the gas-phase, two limits can be considered, depending on the relative rates for thermal "intrasystem" equilibration within the $s$ (including $s^{+}$) and 1 (including $\left.1^{+}\right)$systems, and for the "intersystem" crossing rates between $s^{+}$and $1^{+}$, In the fast intrasystem equilibration limit, the population of each set of transition states is given by the thermal distribution appropriate to the overall s and 1 manifolds. The two reaction rates are then given by the intersystem crossing rates $k_{1+}$ and $k_{s+}$ between the two sets of transition states, weighted by their populations. The net $1-->s$ rate is the difference between these two rates:

$$
\begin{aligned}
& k_{1-\infty}=\left[k_{1+}\left(Z_{1+} / Z_{l}\right) \quad-k_{s+}\left(Z_{s+} / Z_{s}\right)\right] \\
& =\left[k_{1+}\left(e^{-\Delta \vec{H}_{11+} / k T+\Delta S} e^{1+/ k}\right)-k_{s+}\left(e^{-\Delta \bar{H}_{s+} / k T+\Delta S} e e^{/ k}\right)\right] \\
& =\left[k_{1+}\left(e^{-\Delta G_{11+} / k T}\right) \quad-k_{S^{+}}\left(e^{-\Delta G} S^{+/ k T}\right)\right]
\end{aligned}
$$

Here, $\Delta G=\Delta \bar{G}_{a b}-\Delta S a b^{\prime} \Delta \bar{H}_{a b}=\bar{H}_{b}-\bar{H}_{\text {a }}$ and $\Delta S=S_{a b}-S$ are Gibb's free energy, enthalpy. and entropy differences, respectively, between states a and $\mathrm{b}$. 
Fig. 1. Schematic of quantum mechanical energy states associated with the initial $s$ and $I$ configurations. The energized $s^{*}$ and $l^{*}$ states are subsets of the $s$ and 1 states; the critical s+ and 1 . transition states which react are in turn subsets of $s^{*}$ and $1^{*}$.

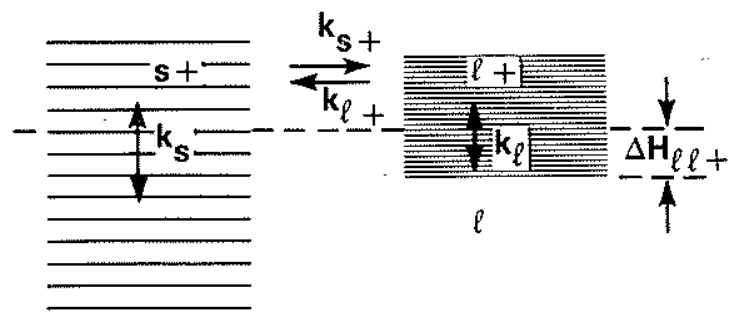

$\mathbf{S}$

\section{LIQUID/SOLID TRANSFORMATIONS (ENTROPY-LIMITED FREEZING)}

Let us now apply this formalism to heterogeneous liquid/solid transformations. The velocity of the liquid/solid interface may be expressed as [15]

$$
v(T)=f \lambda k_{1-->s},
$$

where $f$ is the fraction of interfacial sites at which atomic rearrangement may occur, $\lambda$ is the displacement per rearrangement (the approximate interatomic distance), and $k_{1--}$ is the net liquid to solid rearrangement frequency at active sites, identified with Eqs. (7)-(9) above.

We consider, in particular, the case for which there are large enthalpies and entropies of transformation, e.g., the liquid/solid transformation in silicon. In this case we identify the $s$ and 1 states with solid and liquid, respectively, and suppose that, as drawn in Fig. 1 , the s system has both a lower average enthalpy and a lower entropy (density of states) than the 1 system. Then, it is evident that (a) the average enthalpies of the two transition state manifolds $s+$ and $1+$ are approximately the same, (b) the entropies of the $s^{+}$and $1+$ manifolds are lower than those of the $s$ and 1 manifolds, respectively (since the $s^{+}$and $1+$ manifolds are subsets of the $s$ and 1 manifolds, respectively), and (c) the entropy of the $s^{+}$manifold is much less than that of the $1+$ manifold.

Now, by microscopic reversibility, the intersystem crossing rates between each pair of states within $\mathrm{s}^{+}$and $\mathrm{l}^{+}$must be equal. Therefore the thermally averaged intermixing rates $k_{s+}$ and $k_{1+}$ must be related by the ratio between the partition functions of $s^{+}$and $1^{+}+[16]$ :

$$
k_{S^{+}}=k_{1+}\left(Z_{1+} / Z_{S^{+}}\right)=e^{-\Delta G_{S+1+} / k T} \text {. }
$$

Rewriting Eq. (8) in terms of the larger of the two intersystem crossing rates, $k_{s+}$, then gives

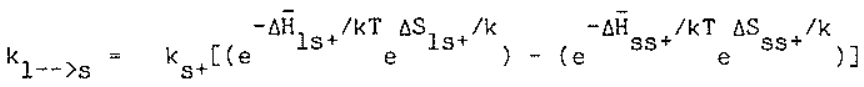

$$
\begin{aligned}
& \left.=k_{S+} e^{-\Delta \bar{H}_{1 S+} / k T} e^{+\Delta S} S^{+} / k\left(e^{-\Delta S} s l^{/ k}\right)-\left(e^{-\Delta \bar{H}_{S I} / k T}\right)\right] .
\end{aligned}
$$

The intersystem crossing rate $k_{s+}$ can be expected to be limited to vibration frequencies. Note that Eqs. (12) and (13) are essentially the same as expressions previously derived by K.A. Jackson [17]. 
Note that if the collisional equilibration rates $k_{s}$ and $k$ are much faster than the intersystem crossing rate $k_{s+}$, a similar expression can be derived, differing slightly in that the prefactor $k_{s^{+}}$must be changed to

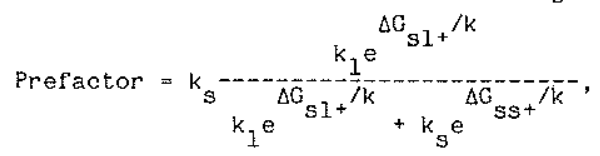

where $k_{s}$ and $k_{l}$ are collisional equilibration rates within the $s$ and 1 systems, respectively. The main difference will be in the magnitudes of $k_{s}$ and $\mathrm{k}_{\mathrm{s}_{+}}$: the "correlation" factor multiplying $\mathrm{k}_{\mathrm{s}}$ in Eq. (14) is less than (but very near) unity, since $\mathrm{S}_{\mathrm{I}^{+}} \gg \mathrm{S}_{\mathrm{S}^{+}}$and hence $\mathrm{G}_{\mathrm{I}^{+}} \gg \mathrm{G}_{\mathrm{S}^{+}}$.

The product of the Boltzmann prefactors appearing in $\mathrm{Eq}$. (13) may be expected to be less than (and perhaps near) unity in magnitude. The remaining expression in brackets represents the difference ketween two terms having only to do with the thermodynamics of bulk solid and bulk liquid. The first term corresponds to freezing, and is decreased from unity by an entropy factor. The second term corresponds to melting, and is decreased from unity by an enthalpic factor.

Because of the entropy factor that enters into the freezing rate, we have previously interpreted Eqs. (10) and (13) as an entropy-limited freezing model: even at very large undercoolings, independent of any additional limitation due to a non-zero activation enthalpy, freezing is limited to rates much lower than collision rates by the difference between the entropies of the solid and the liquid. Physically, such an entropy limitation comes about in the following ways. In the fast intrasystem equilibration limit, the freezing rate is determined by the $1+\cdots s+$ transition rate. Since the $l^{+}$state density is much greater than the $s^{+}$ state density, microcanonical equilibrium demands that the freezing rate be decreased by a large entropy factor. In the fast intersystem crossing Iimit, the freezing rate is determined by the $s+-->s$ transition rate. Since the st and $1+$ states are in microcanonical equilibrium, the population of $\mathrm{s}^{+}$states is low, and, again, the freezing rate will be decreased by an entropy factor.

We would like to acknowledge helpful discussions with P.M. Richards, K.S. Schweizer and M.E. Coltrin.

\section{REFERENCES}

[1] S. Glasstone, K.J. Laidler and H. Eyring, Theory of Rate Processes (McGraw-Hill, New York, 1966); and H. Eyring, S.H. Lin and S.M. Lin, Basic Chemical Kinetics (Wiley-Interscience, New York, 1980).

[2] W. Forst, Theory of Unimolecular Reactions (Academic Press, New York, 1973).

[3] J.W. Christian, The Theory of Transformations in Metals and Alloys, and Ed. (Pergamon Press, Oxford, 1975), Chap. 3.

[4] J.Y. Tsao, M.J. Aziz, M.O. Thompson and P.S. Peercy, Phys. Rev. Lett. 56, 2712 (1986); and manuscript submitted to Phys. Rev. B.

[5] B.C. Larson, J.Z. Tischler and D.M. Mills, J. Mater. Res. 1, 144 (1986).

[6] J.Y. Tsao, P.S. Peercy and M.0. Thompson, J. Mater. Res., to be published.

[7] J.Y. Tsao, P.S. Peercy and M.O. Thompson, unpublished.

[8] It is also possible that transition-state theory alone is inadequate, and that other theories (or combinations of theories), such as those 
suggested by P.H. Bucksbaum and M.O. Thompson (to be published), and by P.M. Richards (to be published), may be necessary.

[9] This is an asymetry additional to that which might already be present due to an activation enthalpy.

[10] F.A. Lindemann, Trans. Faraday Soc. 17, 598 (1922).

[11] In step 1, especially at high pressures, the strong-collision approximation (one-step activation) is usually made: a thermal population distribution is assumed to be established after each collision.

[12] The collisional energization rate into transition states is independent of whether the system is in equilibrium or not, and so is given by the equilibrium rate (which must exactly equal the collisional de-energization rate out of the transition states [2]).

[13] We assume that, in condensed phases under normal atmospheric pressure conditions, enthalpies are approximately equal to energies.

[14] These two assumptions represent important differences between the ideas presented here and those of P.H. Bucksbaum and M.O. Thompson $[8]$.

[15] F. Spaepen and D. Turnbul1, in Laser Annealing of Semiconductors, J.M. Poate and J.W. Mayer, eds. (Academic, New York, 1982), pp. 15-42.

[16] Since $k_{S^{+}}=E^{*} \delta^{\infty}\left[k_{S^{+-}>1+}(E) \rho_{S^{+}}(E) e^{-H / k T} \rho_{1^{+}}(E)\right] d E / z_{S^{+}}$and

$$
\left.k_{1+}=E^{*} f^{\infty}\left[k_{1+-}\right) s^{(E)} \rho_{1+}(E) e^{-H / k T} \rho_{S^{+}}(E)\right] d E / z_{1+},
$$

where $\rho_{g f}(E)$ and $\dot{p}_{f+}(E)$ are the densities of transition states, and $k_{S_{+-}>1+}(\mathrm{E})=\mathrm{k}_{1+->s^{+}}(\mathrm{E})$. Note that the two sets of transition states

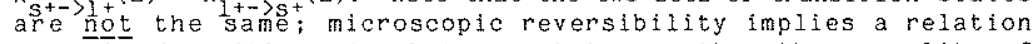
governing transition rates between states, rather than equality of states.

[17] See, e.g., Eq. (9) in K.A. Jackson, in crystal Growth and Characterization. R. Ueda and J.B. Mullin, eds. (North-Holland, Amsterdam, 1975), pp. 21-32. 